\title{
Mass Separation Regimes for Shear-Driven Liquid Film at Expanding Corners
}

\author{
Z. Sadeghizadeh*1, J.A. Drallmeier ${ }^{2}$, J.L. Wegener ${ }^{3}$ \\ ${ }^{1}$ Department of Mechanical Engineering, Florida Polytechnic University, Lakeland, FL,USA \\ ${ }^{2,3}$ Mechanical and Aerospace Engineering Department, Missouri University of Science and \\ Technology, Rolla, MO, USA \\ *Corresponding author email: zsadeghizadeh@floridapoly.edu
}

\begin{abstract}
Separation of gas-driven liquid film from an expanding corner is encountered in many applications such as port fuel injection (PFI) and air-fuel mixing in jet engines. However, physical insight about the liquid mass separation from expanding corners is very limited. Experimental studies show two different flow regimes in shear-driven flows: flow regime where there is no large amplitude waves at the interface and flow regime with large amplitude waves at the interface. Correspondingly liquid mass separation is shown to occur due to two effects: uniform film inertia and large amplitude waves at the interface. In absence of large amplitude waves for large corner angle, the liquid mass separation could occur purely due to uniform film inertia. Two distinct correlations have been proposed for each flow regime based on operating parameters. The controlling parameters, which affect the liquid mass separation at the corner are gas and liquid Reynolds numbers, liquid film properties, and corner angle. Additionally, the proposed correlations would probably need a larger dataset for a robust consistency evaluation.
\end{abstract}

\section{Keywords}

liquid film separation, shear-driven liquid film, Large Amplitude Waves (LAW)

\section{Introduction}

Separation of shear driven liquid film from a sharp corner has applications in many engineering two-phase flow problems. However, there is limited number of experimental and theoretical studies in the literature, which focus on the separation of shear-driven films at sharp corners. The complexity of this problem demands a deep insight into the important physical aspect of the problem in order to establish a comprehensive model for predicting the liquid mass separation. Different approaches are available in the literature to study the liquid mass separation from a sharp corner for different applications.[1] studied the liquid film separation for a PFI engine by visualization techniques. Engine conditions were simulated by adjusting air flow rate at each valve lift to characterize different separation regimes at the valve seat.

In a numerical study presented by [2] the separation of the film was based on the comparison of the film pressure at the corner edge with the pressure developed inside the air flow above the film interface at the same location. In this approach gas pressure was considered as the main factor that controls film detachment from the corner. However, no experimental analysis was conducted to support this hypothesis. In an attempt to define and quantify controlling parameters for liquid film passing through a bend [3] presented a theoretical analysis to model the radial stress distribution that results in film separation from the bend. In this study, a force balance was applied to a control volume of the film turning around a circular bend. Liquid film atomization was predicted by this model depending on the net force exerted on the film at the point of separation. They assume that the liquid interface is smooth and has linear liquid velocity profile. This model was compared to thin films with a thickness less than $0.1 \mathrm{~mm}$ and the accuracy of this model depends on the accuracy of mean film characteristics such as mean film thickness and velocity. Another study[4] presented experimental works to demonstrate that 
mass separation is a strong function of the shearing gas flow and only a weak function of the liquid flow rate which is contrary to other published literature. However, in this experimental study the model was able to predict correctly the onset of the film separation but the prediction of mass separated was limited.

Friedrich el. al [5] developed an analytical model based on momentum conservation for a control volume which includes the liquid ligament. A force ratio term was defined for the ligament control volume to predict the the liquid film separation at the sharp edge. This model was developed to predict the mass separation for thin films in range of $0.1 \mathrm{~mm}<h_{f}<0.5 \mathrm{~mm}$ as a function of mean film properties upstream the corner and it showed a high uncertainty for predicting the onset of film separation from the sharp corner.[6] experimentally studied liquids with different surface tensions and viscosities to demonstrate that while the general behavior of mass separation for each liquid type correlates well to the Friedrich model [5], the model does not provide a reliable quantitative correlation for variation with liquid film properties.

All the models discussed have neglected the effect of the wavy layer at the liquid interface on the liquid mass separation mechanism. Experimental observation in shear driven two-phase flow problems show that when large amplitude waves (LAW) form at the interface, they have high inertia. This large inertial force is due to their high velocities relative to the film substrate velocity and the significant mass content that they carry along the wall ([7], [8], [9], [10], [11]). When these waves encounter a singularity in geometry, they have a tendency to become detached from the corner, which leads to more liquid mass separation. It should be noted that determining the transition from a flow regime without LAW to a flow regime where LAWs are present is still an unsolved challenge in two-phase flow field. In an attempt to establish a mass separation model which considers the effect of LAW at the interface, a force balance model was presented by [12]. However, this model neglected the effect of liquid film properties such as surface tension and viscosity on LAW formation and growth. Furthermore, the inertia due to liquid film substrate was neglected in this study.

The available models in open literature do not capture the complete physics of film separation in a shear-driven flow problem. The purpose of this work is to find a map for liquid mass separation regime of shear-driven liquid films at expanding corners to help in refining existing models. Large amplitude waves(LAW) and liquid film inertia mechanisms are both considered in the impact on liquid mass separation. Experimental results show two distinct liquid mass separation maps, which depend on the LAW existence at the interface. To determine the effective parameters for the empirical correlation for each flow regime, the variation in mean film properties and LAWs at the interface are described physically. The liquid mass separation map correlations are then based on non-dimensional operating conditions, liquid film properties, and the corner geometry.

\section{Experimental Setup}

A schematic of experimental setup is shown in Fig. 1. The facility consists of three sections: air entrance section, test section, and air exit section. The liquid film was driven by the gas flow in $X$ direction and $Y$ axis was defined as normal to the film flow direction. 


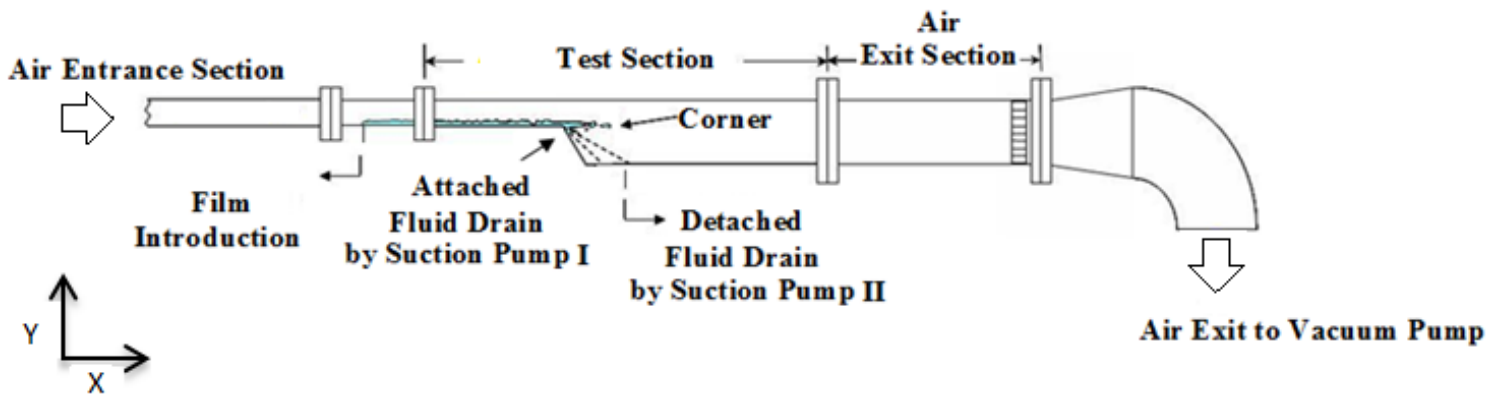

Figure 1. Schematic of experimental unit.

The air entrance region with $1.43 \mathrm{~m}$ length, was designed to provide a fully developed turbulent flow prior to the test section. As is shown in Fig. 2 (a) and (b), two different removable test sections were designed for this study. Fig. 2 (a) with corner angle of $60^{\circ}$, was used to test all flow regimes including both flow conditions with LAWs and without LAWs at the interface. The purpose of designing the right angle corner test section (Fig. 2 (b)) was to study the liquid mass separation due to pure film inertia and in absence of LAWs at the interface.

For each test, the liquid film was introduced in the test section through a porous brass medium.
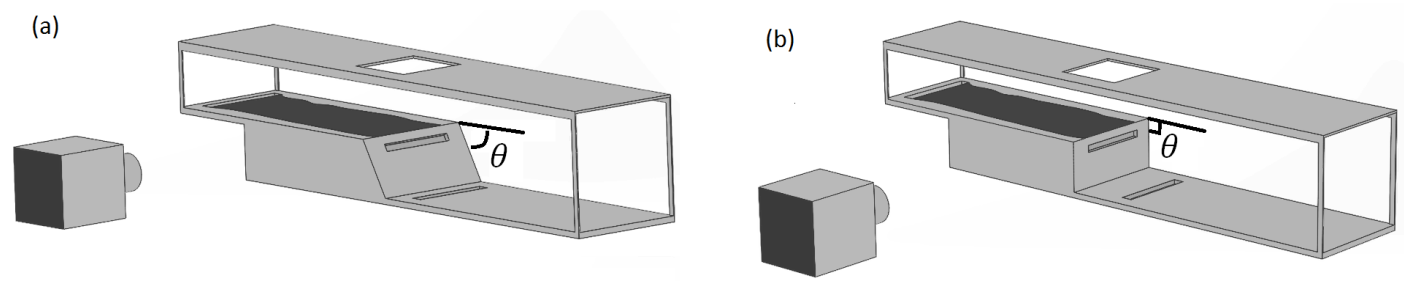

Figure 2. Test sections with different corner angles: (a) $\theta=60^{\circ}$ (b) $\theta=90^{\circ}$.

This location is called film introduction point. The liquid was sent to the brass at the film introduction point from a pressurized vessel. Liquid volume flow rate was adjusted using a rotameter with an uncertainty of $2.5 \%$. A liquid filter was applied before the brass medium to filter any contamination larger than 8 micron in the liquid. Both test sections were designed with a sharp corner, which was located $23 \mathrm{~cm}$ downstream of the film introduction point.

The two brass porous segments, shown in Fig. 3, were implemented on the inclined/vertical surface of the wall right after the corner for each test section to collect the attached liquid, and the other one was located on the lower horizontal wall after the sharp corner to collect the separated liquid. Each brass porous segment was connected to a separate suction pump to collect the attached and detached liquids after the sharp corner without interrupting the separation process.

The cross section before the sharp corner was a rectangle with aspect ratio of 5 (height of $2 \mathrm{~cm}$ and width of $10 \mathrm{~cm}$ ). In order to measure film width nearest to the corner, an optical transparent window was located on the top wall such that optical access was provided $4 \mathrm{~cm}$ upstream from the corner. To have horizontal shear flow, the facility was mounted on an optics table, which provided accurate leveling of the test section in all directions. Also, a high speed camera was used to take images from the side view of liquid film prior to the corner. An image of the test section used in this experiment is shown in Fig. 4.

Section three was the gas exit section, which was connected to a liquid ring vacuum pump to pull the air into the system. A laminar flow element (LFE) was used to measure the air flow rate through the system caused by the vacuum pump. The volumetric flow rate of air was correlated 


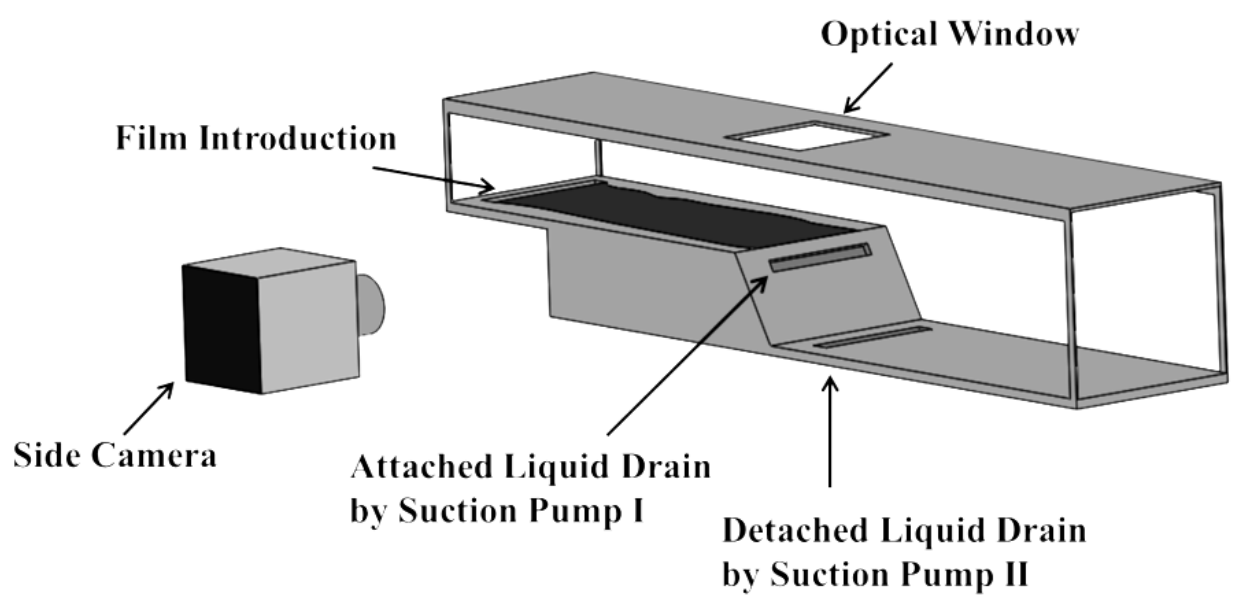

Figure 3. Detailed schematics of the test section.

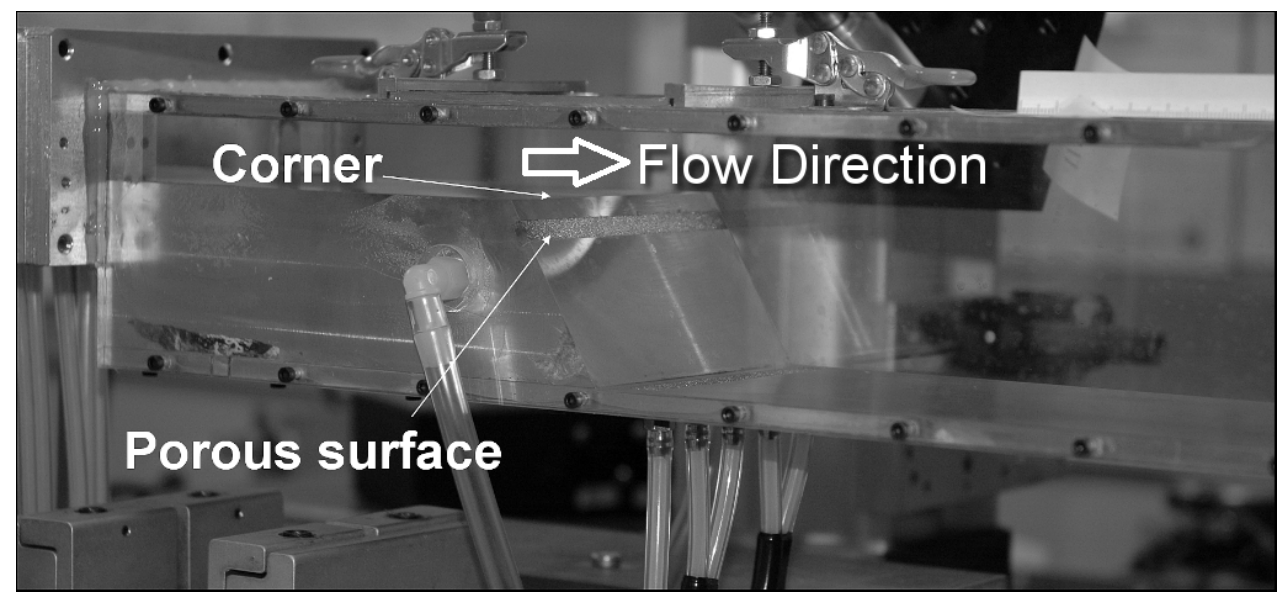

Figure 4. Attached and Separated liquid drainage.

with the pressure drop through the LFE. Having the cross sectional area of the duct, the mean gas velocity was calculated for different flow rates. By adjusting the manual control valve on the vacuum pump, the average gas velocity in this experiment varied from 25 to $40 \mathrm{~m} / \mathrm{s}$. In this study, to have the liquid $R e_{f}$ number, $R e_{f}=\frac{\dot{Q_{f}}}{W_{f} \nu}$ between 70 to 300 , the liquid volumetric flow rate varied from $\dot{Q}_{f}=400 \mathrm{ccm}$ to $\dot{Q}_{f}=1000 \mathrm{ccm}$.

\section{Liquid Matrix}

Three main liquid film properties of density, surface tension, and viscosity impact liquid film mass separation. However, in practice the density variation between commonly studied liquids is not as significant compared to variations in liquid surface tension and viscosity. Therefore, in this paper, only liquid film surface tension and viscosity were considered as liquid film variable properties.

In this experiment, it was found that liquids with surface tensions higher than water $(\sigma=0.072$ $\mathrm{N} / \mathrm{m}$ ) form ridges at the edges of the liquid film due to contact angle effects. Therefore, all liquids used in this study had surface tensions lower than $\sigma=0.072 \mathrm{~N} / \mathrm{m}$.

For the first series of experiments, vinegar ( $5 \%$ acetic acid $\mathrm{CH} 3 \mathrm{COOH}$ by volume), glycerolvinegar mixtures (GV), and butanol-water (BW) mixtures were chosen as the working fluids. In 
Case 1 experiments, vinegar and GV mixtures were used to study the effect of viscosity, since all these mixtures had nearly the same surface tension. In Case 2 experiments, BW mixtures with approximately the same viscosities were prepared to investigate the effect of surface tension on liquid mass separation. Case 1 and Case 2 experiments were performed for the $\theta=60^{\circ}$ test section.

From high speed imaging, gas-liquid flow conditions where no LAWs were visible were determined for Case 3 experiments. For vinegar at all gas velocities and a liquid volume flow rate of $\dot{Q}_{f}=400 \mathrm{~cm}^{3} / \mathrm{min}$, no LAW appeared at the interface. Furthermore, the observations showed that $(10 \% \mathrm{GV})$, and $(20 \% \mathrm{GV})$ with liquid flow rates lower than $\dot{Q}_{f}=600 \mathrm{~cm}^{3} / \mathrm{min}$ and $\dot{Q}_{f}=800$ $\mathrm{cm}^{3} / \mathrm{min}$, respectively, were the operating conditions, where no LAW formed at the interface. Case 3 experiments were performed for these operating conditions with two corner angles of $\theta=60^{\circ}$ and $\theta=90^{\circ}$ to investigate whether the liquid mass separation occurs in absence of LAWs at the interface. All liquid properties measurements have been done using a viscometer and tensiometer, which are shown in Table 1.

Table 1. Experimental liquid matrix.

\begin{tabular}{|c|c|c|c|c|}
\hline Test & Liquid Type & $\rho\left(k g / m^{3}\right)$ & $\mu(c P)$ & $\begin{array}{l}\sigma \\
(m N / m)\end{array}$ \\
\hline \multirow[t]{3}{*}{ Case 1} & Vinegar & 1000 & 1.2 & 58.6 \\
\hline & 10\% Glycerol Vinegar (10\%GV) & 1028 & 1.9 & 60.5 \\
\hline & $20 \%$ Glycerol Vinegar (20\%GV) & 1050 & 3.08 & 58.4 \\
\hline \multirow[t]{3}{*}{ Case 2} & 1\% Butanol Water (1\%BW) & 990 & 1.15 & 60 \\
\hline & $2 \%$ Butanol Water (2\%BW) & 1016 & 1.20 & 49.3 \\
\hline & 4\% Butanol Water (4\%BW) & 1016 & 1.16 & 40.4 \\
\hline \multirow[t]{2}{*}{ Case 3} & 10\% Glycerol Vinegar (10\%GV) & 1028 & 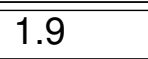 & $\overline{260.5}$ \\
\hline & $20 \%$ Glycerol Vinegar (20\%GV) & 1050 & 3.08 & 58.4 \\
\hline
\end{tabular}

\section{High Speed Imaging Technique}

A high speed side camera (Photron $1280 \mathrm{PCl}$ ) was used in this study to capture high speed images with a shutter speed of 2000 frame per second and a resolution of $640 \times 128$ pixels. Furthermore, the magnification and spatial resolution of these images were 7 and 55 micron, respectively. To determine the interface profile, high speed images were converted into binary sets of data based on pixel brightness. The threshold brightness value of 170 was selected to divide pixels into back and white. This brightness value has been used as a threshold parameter significantly higher than mean brightness value (127.5) to qualitatively distinguishes gaseous and liquid phases at the interface. The pixels with brightness higher than threshold value were converted to 255 , which corresponded to gas phase, and pixels with brightness value lower than 170 were assigned with black pixels(zero brightness), which corresponded to the liquid phase. The interface was determined by the height of the transitional pixels, where the transition between the brightness of 0 and 255 occurred.

In order to visualize the presence of LAWs at the interface, high speed imaging has been used in this study to categorize the flow regimes as: shear-driven without LAW and with LAW. A wave height relative to the mean film thickness ratio of 1.7 was used as a threshold to distinguish ripple waves from LAWs. This assumption is also supported by previous studies. ([13], [14], [7])

Since a line-of-sight effect imposed error on film thickness measurements if determined from the side view, the film thickness was approximated by using a numerical two-phase model presented by [1] rather than experimentally. This 2D numerical model predicted the turbulent air flow field and shear driven liquid film properties, considering the strong interrelated coupling of both phases. Gas-phase flow field characteristics were modeled using a Finite Volume code 
with $k-\epsilon$ turbulent modeling. Due to waviness of the liquid film interface, a special wall function, which was suggested by [15] was considered in this model. It was assumed that gas-liquid film interface was a very slow moving rough wall that could be expressed by equivalent sand grain roughness. Moreover, liquid film propagation was predicted based on a boundary layer description. Typical predicted results are shown in [16].

\section{Analysis}

To determine a liquid mass separation map for different gas-liquid flow regimes, the experimental studies have been divided into two sections. First, the force ratio $(F R)$ analytical model proposed by [5] is used to generate a mass separation map. The $F R$ model only considers uniform liquid film properties and ignores the effect of LAWs on mass separation. To include both effects of uniform film inertia and LAW on mass separation, a new mass separation map is presented in this paper.

\section{Mass Separation Map Based on Force Ratio Model}

An analytical force ratio (FR) model was established by [5] to predict the onset of shear-driven film separation at a sharp corner. This model calculates the force ratio between the forces leading to separation and the forces that resist the separation by using the conservation of linear momentum perpendicular to the ligament at the point of separation.

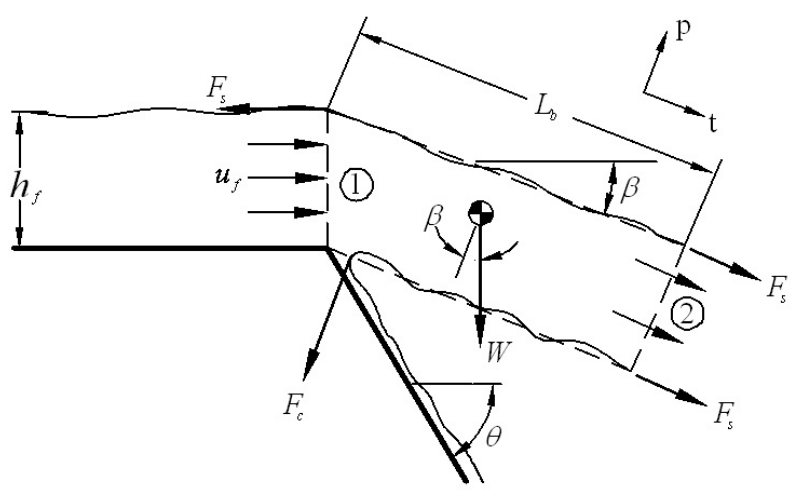

Figure 5. Liquid film at the point of separation.

As it is shown in Fig. 5, for the liquid film two dimensional control volume at the separation point with breakup length of $L_{b}$, the non-dimensional form of $F R$ is written as

$$
F R=\frac{W e_{f}}{1+\frac{1}{\sin \theta}+F r_{h_{f}} W e_{f}\left(\frac{L_{b}}{h_{f}}\right)\left(\frac{1}{\tan (\theta)}\right)}
$$

Where, $W e_{f}=\frac{\rho_{f} U_{f}^{2} h_{f}}{\sigma}$, and $F r_{h_{f}}=\frac{g h_{f}}{U_{f}^{2}}$.

In Eq. $1, \theta$ is the corner angle and $L_{b}$, the estimated length of the film ligament, is estimated from Eq. 2.[17]

$$
L_{b}=0.0388 h_{f}^{0.5} R e_{f}^{0.6} W e_{r e l}^{-0.5}
$$

Here, liquid Reynolds number $R e_{f}$ is

$$
R e_{f}=\frac{h_{f} u_{f} \rho_{f}}{\mu_{f}}
$$

and relative Weber number is defined as

$$
W e_{r e l}=\frac{h_{f} \rho\left(U_{g}-u_{f}\right)^{2}}{2 \sigma}
$$


The effect of liquid film inertia is to separate the film from the corner, while surface tension and gravitational forces inhibit liquid film separation. Also, the $F R$ equal to one corresponds to the onset of liquid film separation. Experimental studies by [6] show that the restoring gravitational force is negligible compared to the surface tension force for sharp corner geometries with large angles.

The liquid film mass separation results versus the $F R$ is depicted in Fig.6. Results have been presented for Case 1 and Case 2 experiments and then compared to previous study results presented by [6], where the laser focus displacement(LFD) technique was used to measure the mean film thickness and consequently mean film velocity to calculate the $F R$ values.

Using LFD measuring unit, a laser source produces a diverging light beam and the optical

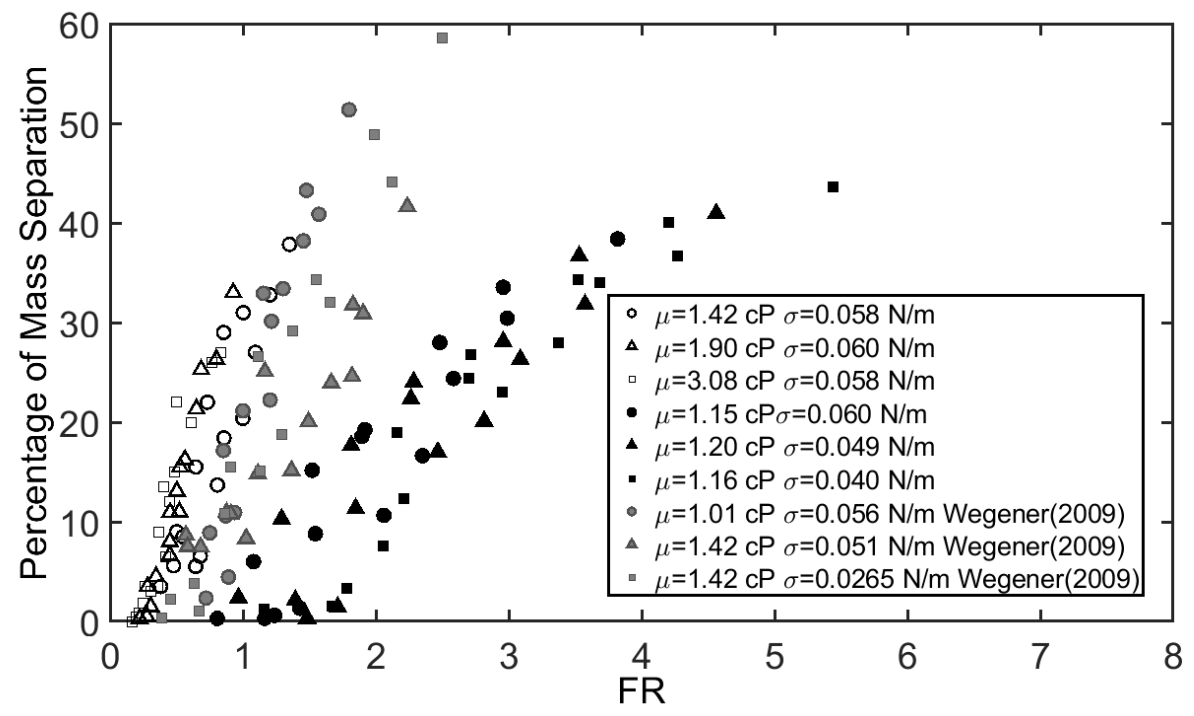

Figure 6. Mass separation versus FR for liquids with different surface tensions and viscosities.

train within the unit applies the confocal principle to detect the location of the interface between the gas and liquid phases. While moving the focal point of a converging laser beam, the LFD instrument locates a surface by sensing peaks in reflected light intensity when the laser's focal point is at the interface of two media. As discussed in experimental setup section, the liquid mass separation results for Case 1 and Case 2 experiments against the $F R$ show two separated trends. For Case 1 experiments the $F R$ values are smaller compared to the Case 2 experiments. Hence, the $F R$ model does not accurately collapse the impact of liquid film surface tension and viscosity into a single trend. Results in Fig. 6 demonstrate that the liquid mass separation can not clearly be explained through the $F R$ model. In the $F R$ model the liquid film structure is simplified as a mean layer with smooth interface. However, in fact the liquid film is a complicated structure consisted of film substrate and wavy layer, which includes a combination of different types of disturbance waves such as ripple waves (capillary waves) and LAWs at the interface. Experimental studies in shear-driven flows show that LAWs carry considerable mass fraction of liquid film and contribute significantly to the liquid mass separation. Having the onset of mass separation at a $F R$ of 0.5 implies that, while the $F R$ correlates to the magnitude of the mass separation, the prediction of mass separation inception is not well captured.

\section{Mass Separation Maps}

Physical criteria that impact the force imbalance at the corner and consequently liquid mass separation were considered to determine a correlation between liquid mass separation and non-dimensional operating parameters. The interaction between the gas phase and liquid film is characterized by $R e_{g} \times R e_{f}$. For air flow in a fixed test section in this study the $R e_{g}$ number 
is equivalent to gas velocity, which is also proportional to mean film velocity in shear-driven flows. ([18], [19], [20]) Hence, for a constant liquid type, $R e_{g} \times R e_{f}$ term represents the liquid film inertia. To consider the effect of liquid film viscosity and surface tension on liquid mass separation, the non-dimensional terms $\frac{\mu}{\mu_{0}}$ and $W e$ number were considered in this correlation, respectively, where $\mu_{0}$ is the viscosity of water. It should be noted that viscosity effect is included in We number through the effect viscosity in mean film characteristics in addition to $R e_{f}$ and $\frac{\mu}{\mu_{0}}$. The liquid film properties impact the mass separation both through influencing mean film characteristics and LAW formation and growth along the $X$ direction. However, the surface tension also influences the liquid mass separation at the point of separation as a restoring force. The corner angle changes the magnitude of the restoring surface tension force relative to the liquid film inertial force, which consequently impacts the force imbalance at the corner. It should be noted that to determine the $R e_{f}$ and $W e$ number, the mean film characteristics were calculated as discussed in experimental setup section. [15]

The liquid mass separation map including all experiments is depicted in Fig.7. White data points refer to the flow conditions in absence of LAWs (Case 3 experiments), which were tested for two corner angels of $\theta=60^{\circ}, 90^{\circ}$ and the black data points are for flow conditions in Case 1 and Case 2 experiments, where LAWs appear at the interface. Although both data sets show the same trend in Fig.7, the maximum liquid mass separation in absence of LAWs is smaller than the maximum liquid mass separation for flow regime with LAWs. Also, the correlation suggests asymptotic behavior at high values for the range of conditions considered in this study. However, broadening the range of experimental conditions to include more breadth in liquid properties and operating conditions still needs to be considered. Since both uniform film inertia and LAW effects are influential on liquid mass separation mechanism, the liquid mass separation map was modified by dividing the map into two flow regimes based on LAW existence.

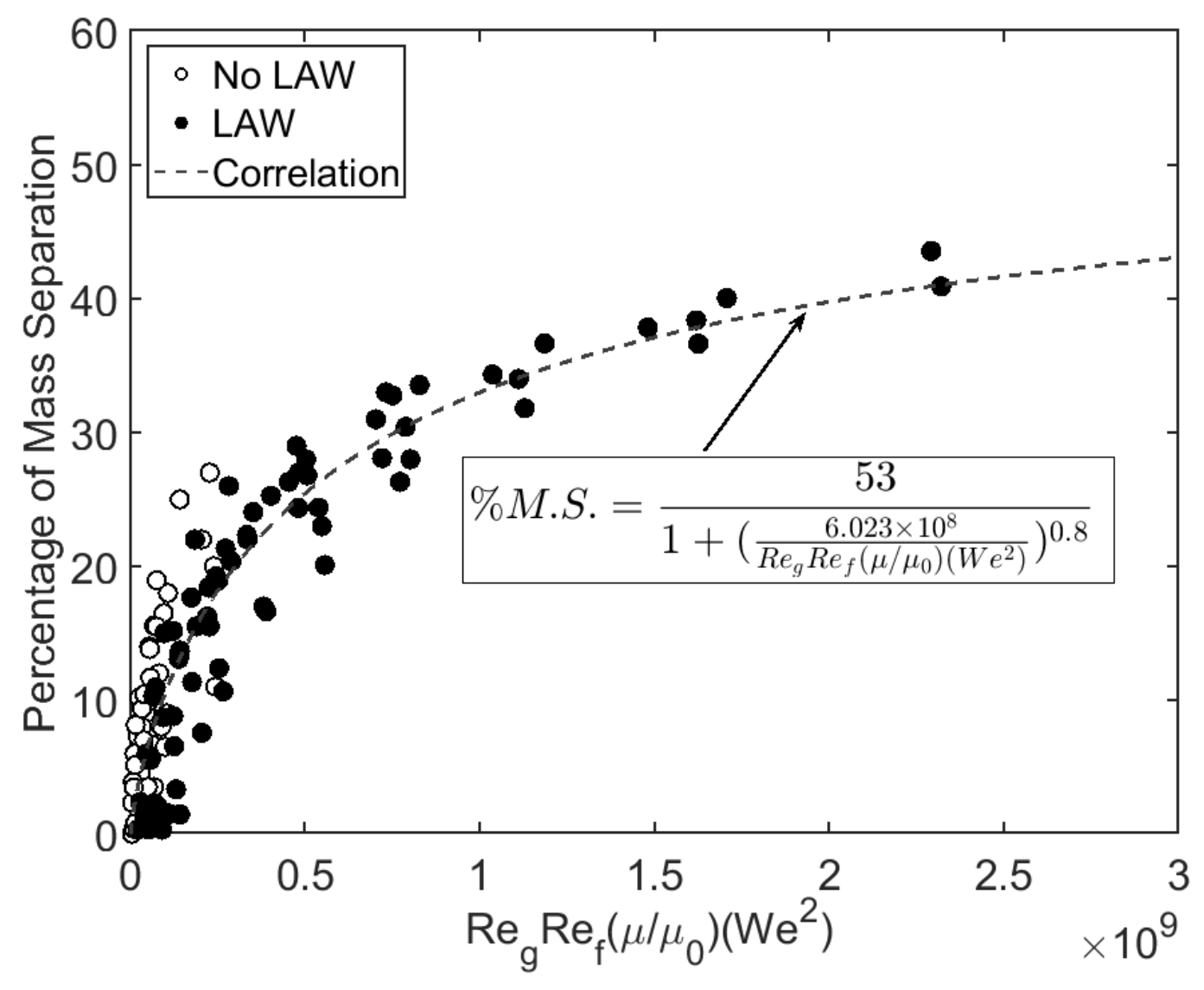

Figure 7. Mass separation map for all flow conditions. 


\section{Prediction of mass separation in presence of LAWs}

All data for the flow regime with LAW are associated to a fixed sharp corner geometry $(\theta=$ $\left.60^{\circ}\right)$. Hence, for specific test section geometry, the non-dimensional parameters that influence liquid mass separation include: $R e_{g} \times R e_{f}$, We number, and normalized viscosity $\frac{\mu}{\mu_{0}}$. These parameters affect the mean film and LAWs characteristics, which impact the force imbalance at the sharp corner.

As is shown in Fig.8, the liquid mass separation correlates well to the defined non-dimensional parameters. In general, the results reveal that liquid mass separation for liquids in Case 1 experiments which have higher surface tension is smaller than liquid mass separation for Case 2 experiments.

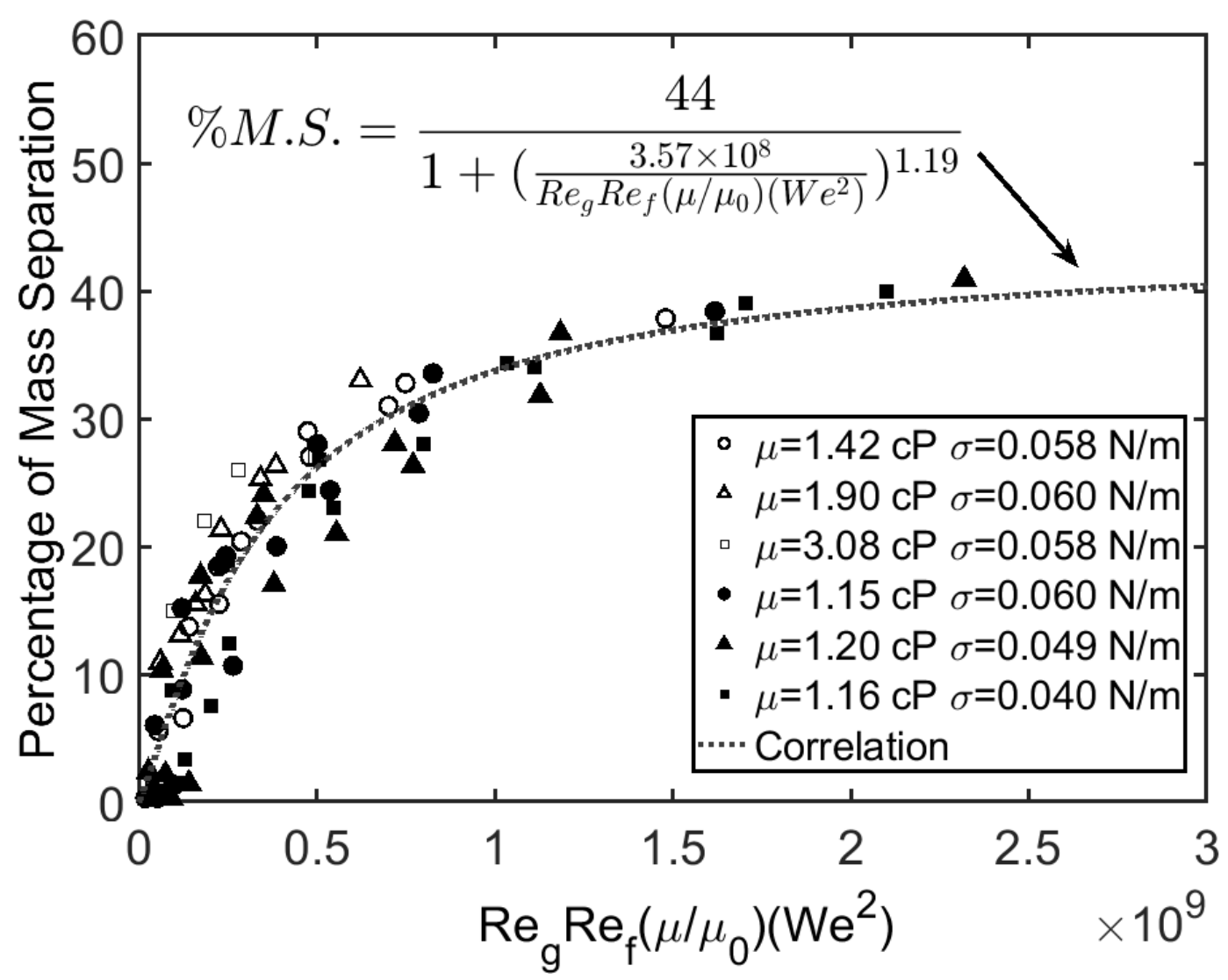

Figure 8. Mass separation correlation for flow regime with LAW.

\section{Prediction of mass separation for flow regime without LAW (Film Inertia Effect)}

Empirical correlation for liquid mass separation in the flow regime without LAW is presented in Fig.9. For the flow regime without LAW considered in this study (Case 3 experiments), the surface tension is approximately constant and does not impact the mean film characteristics and LAW formation and growth along the $\mathrm{X}$ direction. In absence of LAWs at the interface, the driving factor for liquid mass separation is the uniform film inertia. To stimulate the liquid mass separation in absence of LAWs, the force imbalance at the point of separation needs to be increased. Different factors impact the force imbalance at the corner. Since the liquid film properties are coupled with the gas-liquid flow conditions and these conditions were fixed in this experiment, the only remaining parameter for increasing the force imbalance was the corner angle. Therefore, in this experiment, the corner angle was increased from $\theta=60^{\circ}$ to $90^{\circ}$ to increase the force imbalance at the corner in absence of LAWs with the ultimate goal to increase the liquid mass separation.

Therefore, the non-dimensional correlation parameters are: $R e_{g} \times R e_{f}$ and the normalized 


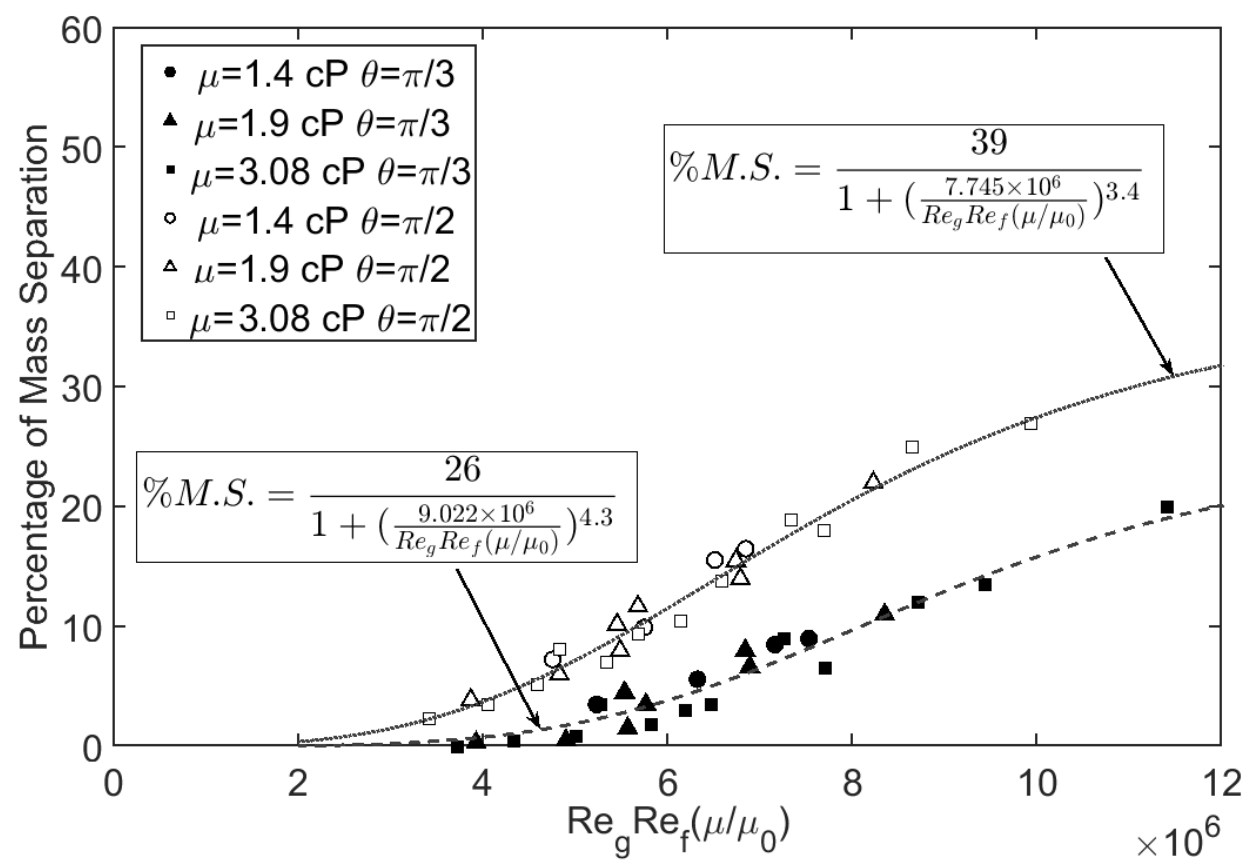

Figure 9. Mass separation correlation for flow regime without LAW.

viscosity $\frac{\mu}{\mu_{0}}$. The non-dimensional parameters in Fig.9 are strongly correlated to liquid mass separation results, which is a better correlation compared to Fig.7. Eliminating $W e$ number from the effective parameters improves the accuracy of the proposed correlation because the We number includes the effect of viscosity through mean film characteristics used to define the We number, which results in scattered data in separation map. A sharp corner with larger angle promotes lower restoring force on liquid film control volume at the corner and leads to higher liquid mass separation for the same operating condition. Comparing the mass separation map in Fig.8 and Fig.9 show that presence of LAWs increase the liquid mass separation. When LAWs with significant mass content reach the sharp corner, they become detached from the corner and lead to a higher percentage of mass separation. This is consistent with previous study presented by [16].

\section{Conclusion}

Two flow regimes exist in applications where shear-driven liquid film forms at the wall: flow regime without LAW and flow regime with LAW. Liquid mass separation is related to inertial force exerted on liquid film. In the case that there is no LAW at the interface, the mass separation occurs entirely due to mean film inertia. For cases where LAWs appear at the interface, the liquid mass separation is driven by inertial force of both mean film and LAWs. For each flow regime, an empirical correlation has been proposed in this study. These correlations were determined based on the physical analysis and high speed imaging observations. Compared to the previous models in literature, these correlations presented the liquid mass separation more accurately as liquid film properties such as surface tension and viscosity are varied. The effect of mean film inertia, LAW formation and growth due to the variation in film properties are captured better in these correlations compare to the previous models, where the effect of liquid film properties on LAWs were completely ignored in mass separation mechanism. It should be noted that the challenge to determine the correlation based on the LAW flow regime is that there is no theoretical approach to identify the transition to LAW flow regime and it is essential to use flow visualization techniques to determine the LAW transition for different flow conditions.Additionally, the proposed correlations would probably need a larger dataset for a robust consistency evaluation. 


\section{Nomenclature}

$Q$ liquid film flow rate $[\mathrm{ccm}]$

$W$ mean film width [mm]

$h$ mean film thickness [ $\mu \mathrm{m}]$

$\sigma$ liquid surface tension $[\mathrm{N} / \mathrm{m}]$

$\mu$ liquid viscosity [cP]

$W e$ Weber number

$R e_{f}$ liquid Re number

$R e_{f}$ liquid Re number

$\theta$ corner angle

$\%$ M.S. percentage of liquid mass separation

$F R$ force ratio

Subscripts

$g$ gas

$f$ liquid

\section{References}

[1] YP Wang, GB Wilkinson, and JA Drallmeier. Experiments in Fluids, 37(3):385-398, 2004.

[2] PJ O'rourke and AA Amsden. A particle numerical model for wall film dynamics in portinjected engines. Technical report, SAE Technical Paper, 1996.

[3] I Owen and DJ Ryley. International Journal of Multiphase Flow, 11(1):51-62, 1985.

[4] BC Steinhaus, JB Ghandhi, and TA Shedd. ILASS Americas, Proceedings of the 20th Annual Conference on Liquid Atomization and Spray Systems, 2007.

[5] Mark A Friedrich, Hai Lan, JL Wegener, JA Drallmeier, and Bassem F Armaly. Journal of Fluids Engineering, 130(5):051301, 2008.

[6] J.L. Wegener. Experiments and Modeling of Shear-Driven Film Separation. MS Thesis, Missouri University of Science and Technology, Rolla, MO, 2009.

[7] TJ Hanratty. Waves on Fluid Interfaces, 11(1):221-259, 1983.

[8] Donald E Woodmansee and Thomas J Hanratty. Chemical Engineering Science, 24(2):299-307, 1969.

[9] N Andritsos and TJ Hanratty. International Journal of Multiphase Flow, 13(5):583-603, 1987.

[10] Kenneth Bruno and MJ McCready. AIChE journal, 34(9):1431-1440, 1988.

[11] Yujie Zhao, Christos N Markides, Omar K Matar, and Geoffrey F Hewitt. International Journal of Multiphase Flow, 55:111-129, 2013.

[12] Evangelos Bacharoudis, Hervé Bratec, Laurent Keirsbulck, Jean-Marie Buchlin, and Larbi Labraga. International Journal of Multiphase Flow, 58:325-337, 2014.

[13] Hideo Nakamura. Research/Japan atomic energy research institute (Tokyo), 96:022, 1996.

[14] Yanzhi Zhang, Ming Jia, Huiquan Duan, Pengfei Wang, Jianxiang Wang, Hong Liu, and MaoZhao Xie. Experimental and numerical study of the liquid film separation and atomization at expanding corners. Technical report, SAE Technical Paper, 2017.

[15] S Wittig, J Himmelsbach, B Noll, HJ Feld, and W Samenfink. ASME 1991 International Gas Turbine and Aeroengine Congress and Exposition, pp. V003T06A017-V003T06A017. American Society of Mechanical Engineers, 1991.

[16] Zahra Sadeghizadeh and James A Drallmeier. Journal of Fluids Engineering, 140(8):081301, 2018. 
[17] T. Arai and H. Hashimoto. Heat Fluid Flow, 20:507-512, 1985.

[18] NS Hall Taylor, IJ Hewitt, JR Ockendon, and TP Witelski. International Journal of Multiphase Flow, 66:38-45, 2014.

[19] K O'Malley, AD Fitt, TV Jones, JR Ockendon, and P Wilmott. Journal of fluid mechanics, 222:139-155, 1991.

[20] N Riley. Journal of engineering mathematics, 21(4):349-361, 1987. 